\title{
Electromagnetic Theory of the Nuclear Interaction
}

\author{
Bernard Schaeffer \\ 7 Rue de l'Ambroisie, Paris, France \\ Email: bschaeffer@wanadoo.fr
}

How to cite this paper: Schaeffer, B. (2016) Electromagnetic Theory of the Nuclear Interaction. World Journal of Nuclear Science and Technology, 6, 199-205. http://dx.doi.org/10.4236/winst.2016.64021

Received: August 3, 2016

Accepted: September 18, 2016

Published: September 21, 2016

Copyright $\odot 2016$ by author and Scientific Research Publishing Inc. This work is licensed under the Creative Commons Attribution International License (CC BY 4.0).

http://creativecommons.org/licenses/by/4.0/

\begin{abstract}
After one century of nuclear physics, its underlying fundamental laws remain a puzzle. Rutherford scattering is well known to be electric at low kinetic energy. Nobody noticed that the Rutherford scattering formula works also at high kinetic energy, needing only to replace the repulsive electric -2 exponent by the also repulsive magnetic -6 exponent. A proton attracts a not so neutral neutron as amber attracts dust. The nucleons have magnetic moments that interact as magnets, equilibrating statically the electric attraction between a proton and a not so neutral neutron. In this paper, the electromagnetic potential energies of the deuteron ${ }^{2} \mathrm{H}$ and the $\alpha$ particle ${ }^{4} \mathrm{He}$ have been calculated statically, using only electromagnetic fundamental laws and constants. Nuclear scattering and binding energy are both electromagnetic.
\end{abstract}

\section{Keywords}

Electromagnetic Interaction, Coulomb, Poisson, Potential, Potential Energy, Neutron, Proton, Deuteron, Helium, Alpha Particle, Nuclear Energy, Nuclear Interaction, Quarks, Strong Nuclear Force, Rutherford Scattering, Anomalous Scattering, Magnetic Moments

\section{Introduction}

Two millenaries ago, Thales discovered the electrical properties of amber $(\epsilon \lambda \epsilon \kappa \tau \rho \emptyset v$, elektron) and the magnetic properties of magnetite (from mount Magnetos) quantified by Coulomb [1] and Poisson [2]. In 1924, Bieler [3] made an unsuccessful attempt to explain with an attractive magnetic inverse fourth-power term in the law of force, unfortunately with the wrong sign. Indeed, it needs only to reuse the Rutherford formula where -2 electric is replaced by -6 magnetic [4].

The neutron, discovered in 1931 by Chadwick, seeming to be uncharged, the electromagnetic hypothesis of the nuclear interaction was abandoned. The magnetic moments of the proton and of the deuteron were discovered in 1932 by Stern [5] and the magnetic moment of the neutron in 1938 by Bloch [6]. Assuming the additivity of the 
magnetic moments, he found that, in the deuteron, the magnetic moment of the neutron was opposite to that of the proton. The existence of a magnetic moment in the neutron proved the existence of electric charges and currents in the neutron, indicating that it is not an elementary particle, as it carries no net charge but still interacts with a magnetic field [6].

In nuclear physics, electric (except so-called Coulomb force) and magnetic interactions between nucleons are still considered to be negligible, although: "The positive charge attracts negative charges to the side closer to itself and leaves positive charges on the surface of the far side. The attraction by the negative charges exceeds the repulsion from the positive charges, there is a net attraction" [7]. Indeed, a proton attracts a neutron as a rubbed plastic pen attracts small pieces of paper.

The electrostatic attraction in the deuteron between a proton and a not so neutral neutron is equilibrated statically by the repulsion between the opposite magnetic moments of the proton and of the neutron. The magnetic interaction between nucleons is attractive or repulsive depending on the position and orientation of their magnetic moments. First theoretical results have been obtained for hydrogen and helium isotopes [8] [9]. The results shown in this paper are for the deuteron and the $\alpha$ particle.

\section{Electromagnetic Potential Energy}

The sum of the electric and magnetic potential energies between electromagnetic particles is the fundamental combination of Coulomb electric and Poisson magnetic potentials [10]-[12]:

$$
U_{e m}=\sum_{i} \sum_{i \neq j} \frac{q_{i} q_{j}}{4 \pi \epsilon_{0} r_{i j}}+\sum_{i} \sum_{i \neq j} \frac{\mu_{0}}{4 \pi r_{i j}^{3}}\left[\boldsymbol{\mu}_{i} \cdot \boldsymbol{\mu}_{j}-\frac{3\left(\boldsymbol{\mu}_{i} \cdot \boldsymbol{r}_{i j}\right)\left(\boldsymbol{\mu}_{j} \cdot \boldsymbol{r}_{i j}\right)}{r_{i j}^{2}}\right]
$$

The first term is the sum of the electrostatic interaction potential energy between electric charges $e_{i}$ and $e_{j}$ separated by $r_{i j}$. The second term is the magnetic interaction potential energy between magnetic moments $\boldsymbol{\mu}_{i}$ and $\boldsymbol{\mu}_{j}$, separated by $\boldsymbol{r}_{i j}$.

\section{Deuteron Electromagnetic Potential Energy}

\subsection{Deuteron Electrostatic Potential Energy per Nucleon (Coulomb)}

The electrostatic potential energy per nucleon $U_{e}$ of this system of the three point charges of the deuteron is, from formula (1), where $\mathrm{A}$ is the atomic number:

$$
\frac{U_{e}^{2} \mathrm{H}}{A}=\frac{1}{A} \times \frac{1}{4 \pi \epsilon_{0}}\left(\frac{q_{1} q_{2}}{r_{12}}+\frac{q_{2} q_{3}}{r_{23}}+\frac{q_{3} q_{1}}{r_{31}}\right)
$$

$q_{1}, q_{2}$ and $q_{3}$ are the three electrostatic charges. $r_{12}, r_{23}$ and $r_{31}$ are their separation distances along their common axis. The total electrostatic potential energy between the 3 electric charges of the deuteron (Equation (2)) becomes fundamental, except for a, adjusted to obtain a horizontal inflection point, replacing a real minimum $\left(r_{n p}\right.$ and $a$ are defined on Figure 1). The electric potential, being negative, is attractive: 


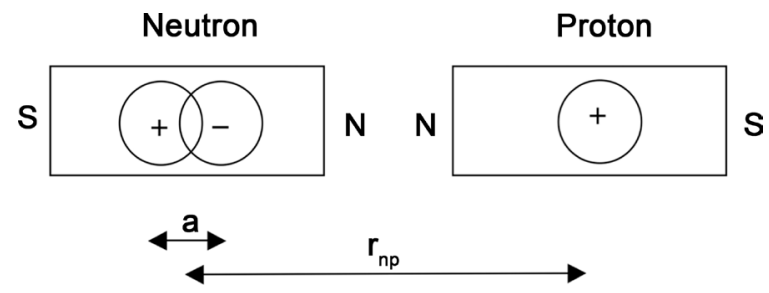

Figure 1. Schematic deuteron structure. The punctual proton contains an electrostatic elementary charge $+e$. The neutron contains electrostatic charges with no net charge, assumed to be $+e$ and $-e$, the elementary electric charges (no proof of the quarks existence found). The electrostatic field of the proton produces the 2 a dipole separation distance between the two opposite electrostatic charges of the not so neutral neutron, distant by $r_{n p}+a$ and by $r_{n p}-a$ from the proton center. The proton attracts electrically the neutron as amber (elektron) attracts dust. The magnetic moments of the proton and of the neutron are known to be collinear and opposite, North against North (or South against South). The magnetic repulsion equilibrates statically the electrostatic attraction. The nuclear binding energies of simple nuclei such as ${ }^{2} \mathrm{H}$ and ${ }^{4} \mathrm{He}$ have been calculated for the first time by applying electromagnetic Coulomb and Poisson fundamental laws.

$$
\frac{U_{e}^{2} \mathrm{H}}{A}=\frac{1}{A} \times \frac{e^{2}}{4 \pi \epsilon_{0}}\left(\frac{1}{r_{n p}+a}-\frac{1}{r_{n p}-a}-\frac{1}{2 a}\right)<0
$$

\subsection{Deuteron Magnetic Potential Energy per Nucleon (Poisson)}

According to the general formula (1) the total magnetic potential energy of the deuteron is:

$$
\frac{U_{m}^{2} \mathrm{H}}{A}=\frac{\mu_{0}}{4 \pi r_{n p}^{3} A}\left[\boldsymbol{\mu}_{n} \cdot \boldsymbol{\mu}_{p}-\frac{3\left(\boldsymbol{\mu}_{n} \cdot \boldsymbol{r}_{n p}\right)\left(\boldsymbol{\mu}_{p} \cdot \boldsymbol{r}_{n p}\right)}{r_{n p}^{2}}\right]
$$

The magnetic moments of the proton and of the neutron in the deuteron are known to be collinear and opposite, $\left(\mu_{n}<0\right.$ and $\left.\mu_{p}>0\right)$, as shown on Figure 1. The coefficient in the brackets above being equal to $-1-(-3)=+2$, the magnetic potential is positive, repulsive:

$$
\frac{U_{m}{ }^{2} \mathrm{H}}{A}=\frac{-2}{A} \times \frac{\mu_{n} \cdot \boldsymbol{\mu}_{p}}{4 \pi r_{n p}^{3}}>0
$$

\subsection{Deuteron Electromagnetic Potential Energy per Nucleon}

Adding the attractive electrostatic Equation (3) and the repulsive magnetic Equation (5), the electromagnetic potential formula (1) becomes, per nucleon, with $A=2$ of the deuteron ${ }^{2} \mathrm{H}$ :

$$
\frac{U_{e m}^{2} \mathrm{H}}{A}=\frac{1}{2} \times \frac{e^{2}}{4 \pi \epsilon_{0}}\left(\frac{1}{r_{n p}+a}-\frac{1}{r_{n p}-a}-\frac{1}{2 a}\right)+\frac{\mu_{0}\left|\mu_{n}\right| \mu_{p}}{4 \pi r_{n p}^{3}}
$$

or, numerically (see Appendix):

$$
\frac{U_{e m}^{2} \mathrm{H}}{A}=0.72\left(\frac{1}{r_{n p}+a}-\frac{1}{r_{n p}-a}-\frac{1}{2 a}\right)+\frac{0.085}{r_{n p}^{3}} \mathrm{MeV}
$$


There is one variable $r_{n p}$ and one parameter $a$ in formula (7). In order to find the binding energy it is necessary to adjust the parameter $a$ of the curve to obtain a potential minimum (in fact, a local minimum, a horizontal inflection point) for both $r_{n p}$ and $a$. This is not to be confused with fitting to adjust the binding energy. A real minimum [13] would be better, of course, but, needing an empirical parameter, would break the fundamental nature of the theory. The horizontal inflection part of the curve corresponds to the deuteron binding energy. The result, obtained by applying electrostatic Coulomb's law and magnetic Poisson's law with the corresponding fundamental constants, is in compliance with the experimental value of the deuteron binding energy per nucleon $-1.11 \mathrm{MeV}$ (Figure 1). There is only one horizontal inflection point per nucleus, adjusted manually by trial and error (Figure 2). It coincides, as by chance, with the binding energy of the nucleus.

\section{Alpha Particle Electromagnetic Energy per Nucleon}

\subsection{Interactions between Neutrons and between Protons}

The single neutron-neutron and proton-proton bonds, being small in comparison with the 4 neutron-proton bonds, have been neglected provisionally. The electric interactions between protons are surely repulsive. The electric interaction between neutrons is probably weak. The magnetic interactions between neutrons and between protons are assumed to be repulsive. The structure of ${ }^{4} \mathrm{He}$ is shown on Figure 3. The graphical solution (Figure 2) gives, for the $\alpha$ particle, a binding energy per nucleon of $-7.6 \mathrm{MeV}$, stronger than the experimental value, $-7.07 \mathrm{MeV}$, by 10 per cent, due probably to the neglect, in a first approximation of the magnetic repulsion between neutrons and between protons. More precise results should be obtained by taking into account these interactions.

\subsection{Proton-Neutron Interactions Only, Provisionally}

We shall calculate the ${ }^{4} \mathrm{He}$ binding energy per nucleon from the deuteron ${ }^{2} \mathrm{H}$ potential energy, which is, as seen before (Equation (6)):

$$
\frac{U_{e m}^{2 \mathrm{H}}}{A}=\frac{1}{2} \times \frac{e^{2}}{4 \pi \epsilon_{0}}\left(\frac{1}{r_{n p}+a}-\frac{1}{r_{n p}-a}-\frac{1}{2 a}\right)+\frac{\mu_{0}\left|\mu_{n}\right| \mu_{p}}{4 \pi r_{n p}^{3}}
$$

A neutron-proton ${ }^{4} \mathrm{He}$ bond is one total attractive ${ }^{2} \mathrm{H}$ deuteron bond, thus $-2.2 \mathrm{MeV}$ equilibrated by the product of 2 magnetic interactions, inclined by $60^{\circ}$, thus dividing the repulsive magnetic potential binding energy twice by 2 thus by 4 . The magnetic moments of the proton and the neutron, being perpendicular (Figure 3), according to formula (1), the Poisson formula has a coefficient of 3 instead of 2 for the deuteron. The potential energy per nucleon is thus:

$$
\frac{U_{e m}^{4} \mathrm{He}}{A}=2 \times \frac{e^{2}}{4 \pi \epsilon_{0}}\left(\frac{1}{r_{n p}+a}-\frac{1}{r_{n p}-a}-\frac{1}{2 a}\right)+\frac{3}{2} \times \frac{\mu_{0}\left|\mu_{n} \mu_{p}\right|}{4 \pi r_{n p}^{3}}
$$

or, numerically (see Appendix): 


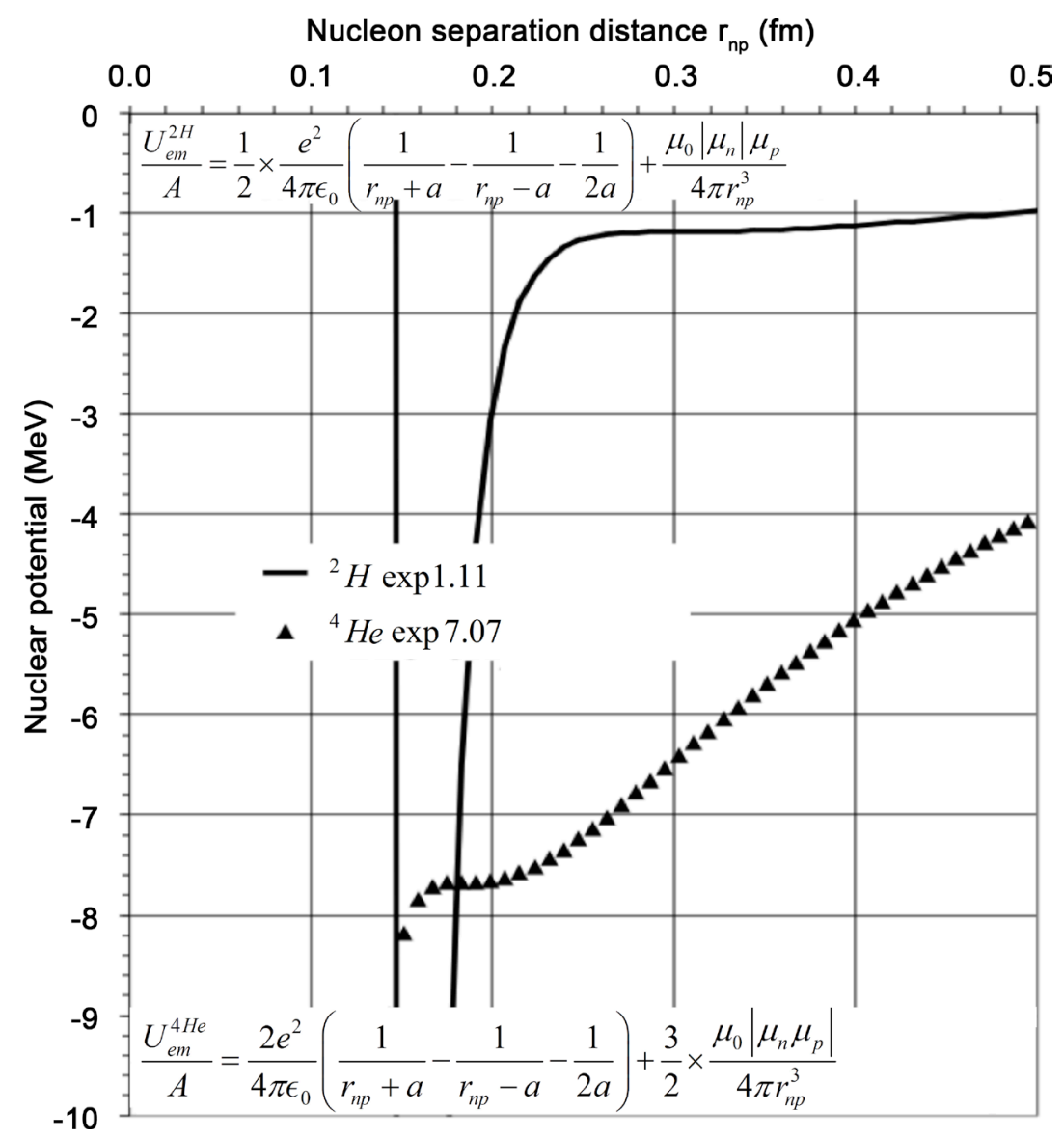

Figure 2. The binding energies per nucleon of ${ }^{2} \mathrm{H}$ and ${ }^{4} \mathrm{He}$ are calculated with Coulomb, electric, and Poisson, magnetic, fundamental laws, without phenomenological parameters. It can be seen that the so-called Coulomb singularity is not a real problem: it just need to replace the minimum by a horizontal inflection point, the radius of the elementary electric charges being unknown, assumed to be point-like. The binding energy is calculated graphically as -1.2 for an experimental value of -1.1 for ${ }^{2} \mathrm{H}$ and $-7.6 \mathrm{MeV}$ for an experimental value of $-7.1 \mathrm{MeV}$ for ${ }^{4} \mathrm{He}$.

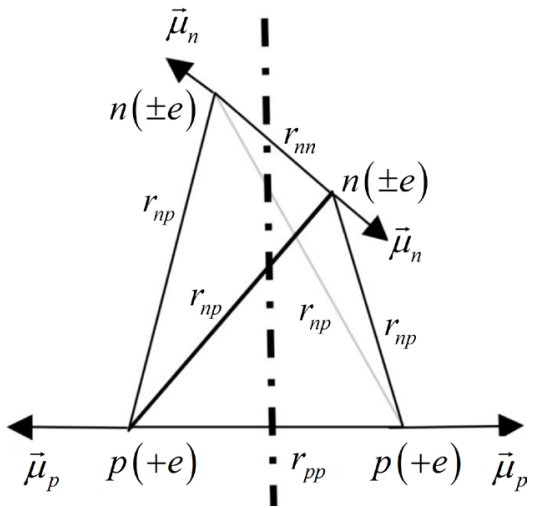

Figure 3. The deuteron ${ }^{2} \mathrm{H}$ has one neutron-proton bond, as shown on Figure 1, thus a total binding energy of $-2.2 \mathrm{MeV}$. ${ }^{4} \mathrm{He}$ has 4 deuteron bonds, inclined at $60^{\circ}$, thus multiplying twice by 4 the total ${ }^{2} \mathrm{H}$ binding energy, giving an approximate total binding energy of $-2.2 \times 4 \times 4=-35 \mathrm{MeV}$ somewhat larger than the experimental value, $-28 \mathrm{MeV}$. The difference is probably due to the repulsive neutron-neutron and proton-proton potentials, not taken into account. 


$$
\frac{U_{e m}^{4} \mathrm{He}}{A}=2.88\left(\frac{1}{r_{n p}+a}-\frac{1}{r_{n p}-a}-\frac{1}{2 a}\right)+\frac{0.128}{r_{n p}^{3}} \mathrm{MeV}
$$

The graphical solution (Figure 2) gives, for the $\alpha$ particle, a binding energy of -7.6 $\mathrm{MeV}$, stronger than the experimental value, $-7.07 \mathrm{MeV}$, too large by 10 per cent, due probably to the magnetic repulsion between neutrons and between protons, neglected in a first approximation. More precise results should be obtained by taking into account these interactions.

\section{Conclusions}

The binding energies of the deuteron and of the $\alpha$ particle have been calculated by applying only fundamental electromagnetic laws and constants with the experimentally proved properties of the nucleons and the nuclei. The binding energy error is about a few percent for the deuteron and almost 10 percent for the $\alpha$ particle, due to the neglect of the neutron-neutron and proton-proton interactions. The only adjusted parameter a is used to obtain the single horizontal inflection point characterizing the binding energy of a nucleus. Not to be confused with fits.

The agreement between experimental results and the electromagnetically calculated Rutherford nuclear scattering (normal and not so "anomalous") and nuclear binding energy proves the electromagnetic nature of the nuclear interaction. No need of hypothetical strong force and quarks.

\section{Acknowledgements}

Thanks to persons at Dubna for their interest to my electromagnetic theory of the nuclear energy. The first question was about scattering. I said I don't know. Now I know: The anomalous Rutherford scattering is magnetic. The second question was: "The strong force doesn't exist?" and a third one about orbiting nucleons [14].

\section{References}

[1] Coulomb, C.A. (1785) Mémoire sur l'électricité et le magnétisme. 2nd Edition, Mémoires de l'Académie Royale des Sciences, Paris.

[2] Poisson (1824) Théorie du magnétisme, Mémoires de l'Académie Royale des Sciences. Paris.

[3] Bieler, E.S. (1924) Large-Angle Scattering of Alpha-Particles by Light Nuclei. Proceedings of the Royal Society of London, Series A, 105, 434-450. http://dx.doi.org/10.1098/rspa.1924.0029

[4] Schaeffer, B. (2016) Anomalous Rutherford Scattering Solved Magnetically. World Journal of Nuclear Science and Technology, 6, 96-102. http://dx.doi.org/10.4236/wjnst.2016.62010

[5] Frisch, R. and Stern, O. (1933) Magnetic Deviation of Hydrogen Molecules and the Magnetic Moment of the Proton. Zeitschrift für Physik, 85, 416.

[6] Bloch, F. (1938) Le moment magnétique du neutron. Annales de I'I.P.H.P., 8, 63-78.

[7] Feynman, R., Leighton, R.B. and Sands, M. (2006) The Electric Dipole. The Feynman Lectures on Physics, 2, 62.

[8] Schaeffer, B. (2011) Electromagnetic Theory of the Binding Energy of the Hydrogen Iso- 
topes. Journal of Fusion Energy, 30, 377-381. http://dx.doi.org/10.1007/s10894-010-9365-0

[9] Schaeffer, B. (2012) Ab Initio Calculation of ${ }^{2} \mathrm{H}$ and ${ }^{4} \mathrm{He}$ Binding Energies. Journal of Modern Physics, 3, 1709-1715. http://dx.doi.org/10.4236/jmp.2012.311210

[10] Owen, G.E. (2003) Introduction to Electromagnetic Theory. Courier Dover Publications, Oxford.

[11] Maxwell, J.C. (1998) A Treatise on Electricity and Magnetism, Vol. 2. Oxford University Press, Great Britton.

[12] Yosida, K. (1996) Theory of Magnetism. Springer-Verlag, Berlin. http://dx.doi.org/10.1007/978-3-662-03297-8

[13] Bobeszko, A. (2015) Electromagnetic Interaction in Deuteron, Potential Energy of Electric and Magnetic Dipoles. http://www.tetrahedralsymmetry.talktalk.net/eid.pdf

[14] Schaeffer, B. (2014) Proton-Neutron Electromagnetic Interaction. ISINN-22, Dubna, 27-30 May. http://isinn.jinr.ru/past-isinns/isinn-22/progr-27_05_2014/Schaeffer.pdf

\section{Appendix: Fundamental Constants Used (CODATA)}

- Light velocity:

$$
c=299 \times 10^{6} \mathrm{~m} / \mathrm{s}
$$

- Fundamental Electric charge

$$
e=1.60 \times 10^{-19} \mathrm{C}
$$

- Electric constant

$$
\frac{e^{2}}{4 \pi \epsilon_{0}}=1.44 \mathrm{MeV} \cdot \mathrm{fm}
$$

- Magnetic constant

$$
\mu_{0}=\frac{c^{2}}{\epsilon_{0}}=4 \pi \times 10^{-7} \mathrm{~N} \cdot \mathrm{A}^{-2}
$$

- Proton mass:

$$
m_{p}=1.67 \times 10^{-27} \mathrm{~kg}
$$

- Proton magnetic moment

$$
\mu_{p}=1.41 \times 10^{-26} \mathrm{~J} \cdot \mathrm{T}^{-1}
$$

- Neutron magnetic moment

$$
\mu_{n}=-0.97 \times 10^{-26} \mathrm{~J} \cdot \mathrm{T}^{-1}
$$

- Proton-neutron magnetic moments combined

$$
\frac{\mu_{0}\left|\mu_{n}\right| \mu_{p}}{4 \pi}=0.085 \mathrm{MeV} \cdot \mathrm{fm}^{3}
$$


Submit or recommend next manuscript to SCIRP and we will provide best service for you:

Accepting pre-submission inquiries through Email, Facebook, LinkedIn, Twitter, etc. A wide selection of journals (inclusive of 9 subjects, more than 200 journals)

Providing 24-hour high-quality service

User-friendly online submission system

Fair and swift peer-review system

Efficient typesetting and proofreading procedure

Display of the result of downloads and visits, as well as the number of cited articles

Maximum dissemination of your research work

Submit your manuscript at: http://papersubmission.scirp.org/

Or contactwjnst@scirp.org 